\title{
MOTOR LEARNING AND TRANSFER BETWEEN REAL AND VIRTUAL ENVIRONMENTS IN YOUNG PEOPLE WITH AUTISM SPECTRUM DISORDER - A PROSPECTIVE RANDOMIZED CROSS OVER CONTROLLED TRIAL.
}

\section{VIRTUAL MOTOR LEARNING IN AUTISM}

Íbis Ariana Peña de Moraes ${ }^{1,2^{* *}}$, Carlos Bandeira de Mello Monteiro ${ }^{1,2^{*}}$, Talita Dias da Silva $^{2}$, Thais Massetti ${ }^{2}$, Tânia Brusque Crocetta ${ }^{3}$, Lilian Del Ciello de Menezes ${ }^{2}$, Gilda Pena de Rezende Andrade ${ }^{4}$, Alessandro Hervaldo Nicolai Ré ${ }^{1}$, Helen Dawes ${ }^{5,6}$, Shelly $\mathrm{Coe}^{6}$, Fernando Henrique Magalhães ${ }^{1}$

${ }^{1}$ School of Arts, Sciences and Humanities - University of São Paulo, São Paulo, SP, Brazil.

${ }^{2}$ Post-graduate Programme in Rehabilitation Sciences, Faculty of Medicine, University of São Paulo, São Paulo, SP, Brazil.

${ }^{3}$ Department of Morphology and Physiology, Faculty of Medicine of ABC, Santo André, SP, Brazil.

${ }^{4}$ Integrated psycho-pedagogical support group (GAPI) Special Education School in São Bernardo do Campo, São Paulo, Brazil.

${ }^{5}$ Institute of Nursing and Allied Health Research, Oxford Brookes University, UK

${ }^{6}$ Department of Clinical Neurology, University of Oxford, Oxford, UK.

* co-first autor

Íbis Ariana Peña de Moraes and Carlos Bandeira de Mello Monteiro should be considered joint first author

** Corresponding author

This study was conducted in the School of Arts, Sciences and Humanities, University of São Paulo, SP, Brazil

\section{LAY SUMMARY}

Individuals with autism spectrum disorder are known to have difficulties with learning motor tasks. Considering that performing motor tasks in virtual environment may be an engaging tool as adjuncts to conventional therapies, we aimed to estimate performance in tasks regardless of physical touch. Results showed that participants had more difficulty using the non-touch task, however, virtual training improved performance on the physical (real) task. This result indicates that virtual methods could be a promising therapeutic approach for the ASD population. 


\begin{abstract}
Autism spectrum disorder (ASD) is associated with persistent deficits in social communication and social interaction, including impaired multisensory integration which might negatively impact cognitive and motor skill performance, and hence negatively affect learning of tasks. Considering that tasks in virtual environment may provide an engaging tool as adjuncts to conventional therapies, we set out to compare motor performance between young people with ASD and a typically developing (TD) control group that underwent coincident timing tasks based on Kinect (no physical contact) and on Keyboard (with physical contact) environments. Using a randomized repeated cross-over controlled trial design, fifty young people with ASD and fifty with TD, matched by age and sex were divided into subgroups of 25 people that performed the two first phases of the study (acquisition and retention) on the same device - real or virtual - and then switched to the other device to repeat acquisition and retention phases and finally switched on to a touch screen (transfer phase). Results showed that practice in the virtual task was more difficult (producing more errors), but led to a better performance in the subsequent practice in the real task, with more pronounced improvement in the ASD as compared to the TD group. It can be concluded that the ASD group managed to transfer the practice from a virtual to a real environment, indicating that virtual methods may enhance learning of motor and cognitive skills. A need for further exploration of its effect across a number of tasks and activities is warranted.
\end{abstract}

Key-words: autistic disorder, developmental disabilities, virtual reality, motor skills.

This study was registered in the clinicaltrials.gov data base with number identifier NCT03254992.

\title{
INTRODUCTION
}

Autism spectrum disorder (ASD) has a worldwide prevalence of $1-2 \%$ based on data from the latest large-scale surveys ${ }^{1}$. ASD is characterised by a qualitative deficit in communication and social interaction as well as a series of restricted, stereotyped and repetitive behaviours and interests ${ }^{2}$. According to Hill and Kearley $(2013)^{3}$ the characteristics of ASD typically appear during the first three years of life and the disorder is one of the fastest growing neurobiological conditions in the world. One important 
consequence resulting from ASD is its impact on social functioning, which leads to a wide range of special needs ${ }^{4}$.

As characteristic of the disease, people with ASD have difficulty in information processing and multisensory integration ${ }^{5}$, affecting cognitive ${ }^{6}$ and motor skills ${ }^{7}$, thereby impairing learning ${ }^{8,}$ performance of different tasks and social communication. According to Stevenson et al. $(2014)^{9}$, these difficulties are a consequence of hypo- and/or hyperresponsivity to sensory stimuli. Emerging evidence suggests that people with ASD have selective deficits in integrating information across all sensory modalities (e.g., vision, hearing, touch and proprioception). This wide range of difficulties, which typically present in childhood, can impact the ability of people with ASD to acquire basic and important cognitive and motor skills required for typical lifelong development ${ }^{10}$. Besides the sensorial deficits, motor impairments have been commonly described in children and adolescents with ASD, such as lack of mimicry in abilities, interpersonal synchronization and motor coordination ${ }^{11}$, poor accuracy ${ }^{12}$, impairments in postural control $^{13}$, motor impairments in balance, gait, and coordination as well as autism-specific impairments in praxis/motor planning ${ }^{14}$ and attentional engagement capacity ${ }^{15}$. These findings raise questions regarding development of motor control and proprioception in young people with $\mathrm{ASD}^{11}$ and suggest that new approaches should be considered in order to enhance motor performance.

In order to minimize these motor difficulties, people with ASD are frequently placed in rehabilitation programs, and health care professionals are continuously seeking new methods to enhance engagement in the rehabilitation process. Thus, virtual reality (VR) has been suggested to help the development of motor skills in ASD both by improving reaction and movement time ${ }^{16}$ and simultaneously increasing physical activity ${ }^{17}$. Importantly, VR has emerged as an engaging and effective tool for improving combined motor and cognitive performance. It is also possible that VR can help to teach more complex integrated cognitive/motor tasks such as those involving planning and executive functioning including bus-taking, shopping and driving activities ${ }^{18}$, and can also train social cognition as an essential component of these interactions ${ }^{19}$. Although, there are still some questions about whether the engagement in VR tasks is due to its novelty and motor interaction, studies highlight that motivation and attention are important features of VR systems (besides ease of use) which may contribute to increased interest and involvement in the tasks ${ }^{20,21}$. Moreover, VR may provide a step change to improve acquisition of a number of community skills and may enable learning in a safe controlled environment, 
improving adherence and enjoyment ${ }^{22}$ and allowing the possibility of enhanced transfer to real-life behaviors ${ }^{23-27}$.

The possible mechanism of using a virtual task to train cognitive and motor performance in ASD is based on an ecological approach to perception and action (see James, 1979) $)^{28}$ which suggests for each action, a specific information-movement coupling is mounted which is specific to the tasks and situation (Savelsbergh and Van der Kamp, 2000) ${ }^{29}$. Van der Weel et al. $(1991)^{30}$ and De Mello Monteiro et al. (2014 and 2017) $)^{31,32}$ found that people with disabilities find performing tasks using a physical interface easier than navigating a virtual environment. Since different tasks or situations require the exploration of different sources of information to control movement ${ }^{29}$ there is likely a difference in performing a similar task on a computer compared with a virtual environment ${ }^{33}$. Tactile feedback may interfere with other stimuli ${ }^{34,35}$, whereas virtual tasks use abstract and intangible interactions. It is therefore hypothesised that performing a task in a virtual environment may result in impaired performance in young people with ASD due to lack of tactile feedback. However, based on the study by Martins et al. $(2016)^{36}$ the difficulty experienced in a virtual environment may create new motor strategies, enhancing the transfer of learning to other environments in young people with $\mathrm{ASD}^{32}$.

Considering the possible benefits of using virtual devices, performance and transfer between learning environments were evaluated when young people with ASD and those with Typical Development (TD) used virtual (Kinect system) and real devices (Keyboard or Touch Screen). We set out to estimate performance in Kinect compared to Keyboard environments, and transfer in performance between these environments to a touch screen device.

\section{METHODS}

This study was approved by the research ethics committee of the University of São Paulo (CAAE: 55498016.1.0000.5390) and was registered in the clinicaltrials.gov data base with the following number identifier: NCT03254992. Design: Randomized repeated exposure cross-over controlled trial. 


\section{PARTICIPANTS}

100 people were invited to participate and were classified into one of two groups. The ASD group consisted of 50 people aged between 7 and 15 years $(11.3 \pm 2.4,38$ male and 12 female). The TD group consisted of 50 people matched by age and sex to the ASD group and were recruited from a public school in the city of São Paulo. All participants signed the informed assent form and parents or the legal guardians signed the informed consent form. The ASD group had all previously been evaluated at the Integrated PsychoPedagogical Support Group (GAPI) - Special Education School in São Bernardo do Campo, São Paulo, Brazil, an institution specialised in children and adolescents with invasive developmental disorders.

Inclusion criteria included the following: (1) for the ASD group, people with mild and moderate degrees of autism diagnosed by a child neurologist and were evaluated by by researchers with a patient history, psychological assessment, communication evaluation and psychiatric evaluation; (2) between Grades 1 and 5 of elementary school.

Participants were excluded if they did not understand the task (assessed through the ability of the participant to perform the task correctly after performing three attempts with explanation and demonstration of the evaluator) (14 exclusions), or who withdrew during the study (2 exclusions).

Severity of autism was assessed by Childhood Autism Rating Scale (CARS), classified according to the following: a total score of 15-29.5 was considered 'nonautistic', a score of 30-36.5 was considered 'mild to moderate' autism and a score of 3760 is considered 'moderate to severe' autism ${ }^{37}$. Additionally, a short form of the Wechsler Intelligence Scale for Children (WISC-III) (Campbell 1998) was used as a general intellectual screener for the subgroups with ASD. WISC-III has been shown as a valid measure of general intelligence ${ }^{38}$ and uses the following classification of the Intelligence Quotient (IQ): mild intellectual disability at 55-70, borderline intelligence at $70-85$, normal intelligence at 85 or above, above average intelligence at 115-129, and superior intelligence at 130 or above $^{39}$. Characterization of ASD subgroups as to this measure of general intelligence is shown in the Results section (Table 1).

After inclusion and exclusion criteria, a simple randomization technique by computer-generated random numbers was used. Age, IQ, and CAR scores was continuously checked (at every 10 subjects allocated in each group) to assure homogeneity between the demographics of the groups. 


\section{INSTRUMENT}

\section{Coincident timing task}

For the data collection, a software game was created in partnership with the Information Systems Department at the School of Arts, Sciences and Humanities of University of São Paulo ${ }^{40}$. The game offers a coincident timing task and refers to the ability of the participant to perform a movement where the arrival of the hand (gesture or touching) at a target coincides with the arrival time of a moving object ${ }^{41}$. In the proposal of this work, the game requires that the participant try to move the avatar of his or her hand to coincide with the lighting of the last bubble (target bubble). As previously described by De Mello Monteiro et al. (2014) ${ }^{32}$ during the virtual task, they were instructed to 'intercept' a virtual bubble by doing a manual movement (i.e., a waving gesture) on a non-physical interaction device (Kinect), considered as device A. In the physical task in which the keyboard space button is pressed, participants were instructed to 'intercept' a falling virtual object as it reached the target by pressing a button on the computer (device B) or touching the touchscreen on the computer (device C).

The magnitude and direction of error of each participant in anticipating or delaying the arrival of the light to the target was recorded by the software in milliseconds. The objective was to evaluate the time difference between the execution of the participant's response and the arrival of the object at the target location and the global temporal precision and therefore the coincidence-anticipation ability of time ${ }^{31,32,42}$.

The software was programmed to provide a unique username for each participant where the following data was stored: participant name, date of birth, sex and name of the researcher.

\section{PROCEDURES}

Participants were placed comfortably in a chair that was adjusted according to their size and needs so that they were properly positioned to allow the task to be performed. Before starting, the task was verbally explained and a demonstration was offered by the examiner. The execution time was observed in each experimental stage and explained as follows: acquisition (A), retention $(\mathrm{R})$ and transfer $(\mathrm{T})^{43}$. A short-term motor learning protocol was used based on previous studies by Gidley Larson and Mostofsky $(2008)^{44}$, Torriani-Pasin et al. (2013) ${ }^{45}$ De Mello Monteiro et al. (2014 and 2017) $)^{31,32}$ and Bonuzzi et 
al. $(2017)^{46}$ The motor learning protocol was organised through blocks of five trials each to ensure appropriate and representative evaluation of the participants' performances. All participants took 20 attempts at acquisition (divided into four blocks), five attempts at retention (test was then performed after 5 minutes without contact with task) and five attempts at transfer (performed on task with touch screen). During the attempts, the bubbles simulated a movement of light falling with a total time of 5 seconds between the first and last bubble as proposed by Monteiro et al. $(2014 \text { and 2017) })^{31,32}$.

Participants used three interfaces for the same task: (A) Kinect for windows virtual task (task of gesture, non-physical contact, with a more virtual environment) and (B) Keyboard - real task (task on space button on keyboard, with physical contact and a more real environment), considering acquisition and retention phases, and (C) Touch screen for the final transfer phase. For the transfer phase, a different interface was used due to it being used in the daily life of most of the population. The individuals in each group (ASD and TD) were divided into two sequences (quasi-experimental design, considering that the age and gender difference could influence the results) according to the order of the task: sequence A and sequence B. In sequence A, the task was performed in the virtual environment first by using Kinect followed by the task in the keyboard on acquisition and retention phases. In sequence B, the inverse process was performed, executing Keyboard first and then the Kinect on acquisition and retention phases. For both sequences the touch screen interface was used for transfer phase (Figures1 and 2).

Figure 1. Representative design of the accomplishment of the coincident timing task in the use of the kinect interface.

[figure 1 is here]

A. Demonstration of error performed by the participant on each attempt (red light - unsuccessful). B. Demonstration of hit performed by the participant on each attempt (green light - successful). Main image is about an example of a participant performing the task on Kinect. 1. Performing the task on the touch screen interface. 2. Performing the task on the keyboard interface.

Figure 2. Experimental design.

[figure 2 is here]

TD: typical development; ASD: autism spectrum disorder.

\section{DATA ANALYSIS}

Descriptive statistics; analysis was performed considering the dependent variables age, weight, Height and Body Mass Index. Multiple Analysis of Variance (MANOVA) was carried out in order to find out differences between ASD and TD groups and interface subgroups (A and B), with Least Significance Difference used as the post-hoc test (Table 1). For the Intelligence Quotient and the Childhood Autism Rating Scale, independent 
sample t-tests were used for comparing subgroups A and B. For the variable sex, chisquare test was used in order to compare distribution of males and females between subgroups A and B in both ASD and TD groups.

The dependent variables used were timing errors (i.e., constant error (CE), absolute error (AE) and variable error (VE)). The timing error was defined as the time difference between the time the target bubble switched on (arrival time) and the time that the touch or the gesture was registered. Constant Error is the temporal interval (in milliseconds) between the arrival of the visual stimulus and the end of the participant's motor response, the calculation was done by a simple arithmetic average of the error values, considering the algebraic sign (negative or positive) in a series of attempts and represents the timing of error (if it is late or early). $\mathrm{AE}$ is the measure of performance accuracy, which is the estimator of the probability that an individual responds within a range around a target, as calculated by taking the absolute value of each raw score and disregarding whether the response was early or late. VE is the timing error resulting from within-subject variability. It represents the consistency in a group of responses and is independent of the proximity of each trial to the designated target. The calculation considered the square root of the sum of the square of difference between each score and the individual CE mean divided by the number of trials ${ }^{47}$.

Average blocks of five attempts were used, i.e. four acquisition blocks (A1-A4), one retention block $(R)$ and one transfer block $(T)$. The dependent variables were presented as mean and standard error and were submitted to a 2 (group: ASD, TD - between) by 3 (interface: Kinect, Keyboard, Touchscreen - between) by 2 (sequence: real task first, virtual task first - within) by 2 (block - within) MANOVA with repeated measures on the last two factors. For the factor block, separate comparisons were made for acquisition (first acquisition block A1 versus final acquisition block A4), retention (A4 versus retention block R) and transfer (A4 versus transfer block T). Partial eta- squared $\left(\eta^{2}\right)$ was reported to measure effect size and interpreted as small (effect size $>0.01$ ), medium (effect size $>0.06$ ), or large (effect size $>0.14)^{48}$. Post hoc comparisons were carried out using the LSD (least significance difference) test $(\mathrm{p}<.05)$.

Finally, a regression analysis was performed in order to determine if IQ or CARS influenced on the improvement of performance, with the dependent variable being the difference between the last and first block of practice in both AE and VE ( $\Delta$ A4 - A1). All analyses were performed using SPSS 20.0 software package. 


\section{RESULTS}

\section{DEMOGRAPHICS}

Table 1 shows demographic data and comparisons for each of the subgroups. There were no significant findings for any of the variables assessed.

Young people in the ASD group showed a mild/moderate degree of severity of autism as assessed by CARS and IQs ranging from mild intellectual disability to normal intelligence (Table 1).

Table 1. Demographic data and comparisons for each of the subgroups.

[table 1 is here]

ASD: group with autism spectrum disorder; TD: group with typical development; ASD-A and TD-A: groups that started with Kinect; ASD-B and TD-B: groups that started with Keyboard; M: mean; SD: standard deviation; min: minimum value; max: maximum value; md: mean difference; 1 : lower bound of the Confidence Interval (95\%) of the difference; u: upper bound of the Confidence Interval $(95 \%)$ of the difference; n: sample size; BMI: Body Mass Index; IQ: Intelligence Quotient; CARS: Childhood Autism Rating Scale. *MANOVA was carried out in order to find out differences ASD and TD groups and interface subgroups (A and B) with Least Significance Difference as post-hoc test;** values presented regarding posthoc tests; $\dagger$ independent samples t-test comparing subgroups A and B; §chi-square test, probably the sample size was small to find significant results.

In the following paragraph, results are divided by the phases of the motor learning protocol, i.e. Acquisition, Retention and Transfer (with motor performance measured in terms of $\mathrm{CE}, \mathrm{AE}$ and $\mathrm{VE})$. The values are represented as mean (m), mean difference (md) and Confidence Interval (CI) of the difference.

\section{ACQUISITION-A}

The MANOVA was done considering Constant Error (if movement was late or early), Absolute error (accuracy of movement) and Variable Error (precision of movement). The results revealed a significant effect for the blocks $\left(F_{3,94}=2.9, p=0.039\right.$; Wilks' lambda $=0.915)$. Significant interactions were also found between Interfaces by Sequence $\left(F_{3,94}=7.5, p<0.001 ;\right.$ Wilks' lambda $\left.=0.288\right)$ and Interface by Group by Sequence $\left(F_{3,94}=3.4, p=0.022 ;\right.$ Wilks' lambda $\left.=0.903\right)$.

Main effects were found also for the ASD and TD groups, $\left(\mathrm{F}_{3,94}=8.3, \mathrm{p}<0.001\right.$; Wilks' lambda $=0.791)$ and Sequence, $\left(F_{3,94}=5.4, \mathrm{p}=0.002 ;\right.$ Wilks' lambda $\left.=0.852\right)$. Separate follow-up repeated measures (RM-ANOVAs) for CE, AE and VE are reported in the paragraphs below. 
Figure 3 presents the CEs during acquisition on the Keyboard and Kinect interface tasks among the ASD and TD groups for participants who started the real task first compared to those who started the virtual task first. For the CE, only the effects for the Groups (ASD and TD), Sequence (real task first and virtual task first) and Interfaces (Keyboard and Kinect) were described in order to see the directional trend of movement (delay or anticipation). The ANOVA revealed a significant effect for Sequence $\left(F_{1,96}=\right.$ $13.1, \mathrm{p}<.001, \mathrm{y}^{2}=0.12$ ), showing that all participants performing on either the real or virtual tasks first showed trends of a delay in movement, but the participants that performed on the real task first showed more of a delay $(\mathrm{m}=511 \mathrm{~ms})$ compared with the participants who used virtual task first $(\mathrm{m}=305 \mathrm{~ms}$; md $(\mathrm{CI})=206.4(93.3,319.5)$; $\mathrm{p}<0.001)$. No further effects were found.

Figure 3. Constant error for all phases of the study by both groups, sequences and interfaces (mean and standard error).

[figure 3 is here]

K: kinect; KB: keyboard; TS: touch screen; TD: typical development; ASD: autism spectrum disorder; A: acquisition phase blocks (1 through 4); R: retention phase; T: transfer phase.

\section{Absolute Error- $A E$}

The pattern of AEs is illustrated in Figure 4. Significant effects were found for Blocks $\left(\mathrm{F}_{1,96}=4.6, \mathrm{p}=0.035, \mathrm{y}^{2}=0.05\right)$ and Groups $\left(\mathrm{F}_{1,96}=35.4, \mathrm{p}<0.001, \mathrm{y}^{2}=0.27\right)$. These results mean that all participants from both ASD and TD group improved with practice, decreasing the $\mathrm{AE}$ from $771 \mathrm{~ms}$ on the first block of acquisition to $701 \mathrm{~ms}$ on the last block, and the ASD group presented a larger AE $(\mathrm{m}=872 \mathrm{~ms})$ than the TD group $(\mathrm{m}=$ $600 \mathrm{~ms})$. Interaction was found for Interface by Sequence, $F(1,96)=211.3, p<.001, \mathrm{y}^{2}=$ .69. The post hoc comparisons showed that in both sequences, the AE was higher for the Kinect interface (i.e. virtual task first: Kinect interface: $\mathrm{m}=1004 \mathrm{~ms}$ and Keyboard interface: $\mathrm{m}=400 \mathrm{~ms}$; $\mathrm{md}(\mathrm{CI})=603.7$ (496.3, 711.1); $\mathrm{p}<0.001 /$ real task first: Keyboard interface: $\mathrm{m}=515 \mathrm{~ms}$ and Kinect interface: $\mathrm{m}=1024 \mathrm{~ms}$; $\mathrm{md}(\mathrm{CI})=-508.8(-616.2,-401.4)$; $\mathrm{p}<0.001)$.

In order to compare the performance on Keyboard considering the abstract task first, a Student's t-test was used which found that the individuals who started on Keyboard 
had worse performance on acquisition blocks A1 and A4 $(\mathrm{m}=515 \mathrm{~ms})$ compared to the group who started on the Kinect and then performed the task on Keyboard $(\mathrm{m}=400 \mathrm{~ms}$; $\mathrm{p}<0.001)$. This result suggests that using a virtual task can benefit the performance in a real task in both ASD and those with TD.

Figure 4. Absolute error for all phases of the study by both groups, sequences and interfaces (mean and standard error).

[figure 4 is here]

K: kinect; KB: keyboard; TS: touch screen; TD: typical development; ASD: autism spectrum disorder; A: acquisition phase blocks (1 through 4); R: retention phase; T: transfer phase.

\section{Variable Error-VE}

The VE during acquisition is depicted in Figure 5. ANOVA revealed significant main effects by Block, $\left(\mathrm{F}_{1,96}=5.2, \mathrm{p}=0.024, \mathrm{y}^{2}=0.05\right)$ and Group, $\left(\mathrm{F}_{1,96}=23.4, \mathrm{p}<\right.$ $\left.0.001, \mathrm{y}^{2}=0.20\right)$. However, these factors did significantly interact as attested by significant effects for Block by Group $\left(\mathrm{F}_{1,96}=4.4, \mathrm{p}=0.039, \mathrm{y}^{2}=0.04\right)$. Post hoc comparisons indicated that the ASD group had a significantly decreased VE with practice from A1 to A4 $(\mathrm{m}=816 \mathrm{~ms}$ to $614 \mathrm{~ms}$; $\mathrm{md}(\mathrm{CI})=202.2(72.7,331.7) ; \mathrm{p}=0.003)$, while for TD group this difference did not occur $(\mathrm{m}=394 \mathrm{~ms}$ to $385 \mathrm{~ms}$; $\mathrm{md}(\mathrm{CI})=(72.7,331.7) ; \mathrm{p}=0.003)$.

Results showed interaction for Interface by Sequence. Similarly to AE, the post hoc test for VE showed that for both sequences, the VE was higher for the Kinect interface (i.e. virtual task first: Kinect interface: $\mathrm{m}=874 \mathrm{~ms}$, and Keyboard interface: $\mathrm{m}=294 \mathrm{~ms}$; $\mathrm{md}(\mathrm{CI})$ $=580.7$ (438.9, 722.5); $<<0.001 /$ real task first: Keyboard interface: $\mathrm{m}=293 \mathrm{~ms}$, and Kinect interface: $\mathrm{m}=748 \mathrm{~ms} ; \mathrm{md}(\mathrm{CI})=-454.9(-313.1,-596.7) ; \mathrm{p}<0.001)$.

Figure 5. Variable error for all phases of the study on both groups, sequences, and interfaces (mean and standard error).

[figure 5 is here]

K: kinect; KB: keyboard; TS: touch screen; TD: typical development; ASD: autism spectrum disorder; A: acquisition phase blocks (1 through 4$)$; R: retention phase; T: transfer phase.

\section{RETENTION - R}

Figures 3, 4 and 5 also present the timing errors during acquisition and retention regarding Constant Error (if they had anticipated or delayed movement), Absolute error (accuracy of movement) and Variable Error (precision of movement). No distinct differences in the pattern of errors in the final acquisition and retention blocks were 
apparent. Accordingly, MANOVA and ANOVAs did not reveal any significant main effects or interaction when comparing the final acquisition block A4 and the retention block $\mathrm{R}$ for the constant, absolute and variable error.

However, a significant main effect for Group was found for $\mathrm{AE}\left(\mathrm{F}_{1,96}=24.1, \mathrm{p}<\right.$ $\left.0.001, \mathrm{y}^{2}=0.20\right)$. The ASD group presented a larger Absolute Error (AE) $(\mathrm{m}=820 \mathrm{~ms})$ compared with the TD group $(\mathrm{m}=582 \mathrm{~ms})$. For Variable Error (VE), an effect for Group was also found $\left(\mathrm{F}_{1,96}=15.1, \mathrm{p}<0.001, \mathrm{y}^{2}=0.14\right)$, and similarly to the AE, the ASD group had a larger VE $(\mathrm{m}=605 \mathrm{~ms})$ than the TD group $(\mathrm{m}=368 \mathrm{~ms})$. In addition, considering CE all participants maintained a directional trend for delayed movement.

\section{TRANSFER- T}

The MANOVA was performed considering Constant Error (directional trend, if movement was late or early), Absolute error (accuracy of movement) and Variable Error (precision of movement). The results revealed significant effects for Group $\left(F_{3,94}=12.6, p\right.$ $<0.001 ;$ Wilks' lambda $=0.713)$, Sequence $\left(F_{3,94}=17.9, \mathrm{p}<0.001 ;\right.$ Wilks' lambda $=$ $0.635)$ and Interface, $\left(\mathrm{F}_{3,94}=3.8, \mathrm{p}=0.013\right.$; Wilks' lambda $\left.=0.893\right)$. Significant interactions were also found for Block by Sequence, $\left(\mathrm{F}_{3,94}=8.7, \mathrm{p}<0.001\right.$; Wilks' lambda $=0.783)$, Block by Group, $\left(F_{3,94}=2.6, p=0.05\right.$; Wilks' lambda $\left.=0.923\right)$ and Interface by Block by Sequence $\left(\mathrm{F}_{3,94}=64.9, \mathrm{p}<0.001\right.$; Wilks' lambda $\left.=0.326\right)$. Separate follow-up RM-ANOVA's for CE, AE and VE are reported in the paragraphs below.

\section{Constant Error - CE}

The results for $\mathrm{CE}$ showed a significant effect for Sequence $\left(\mathrm{F}_{1,96}=21.1, \mathrm{p}<\right.$ $\left.0.001, \mathrm{y}^{2}=0.18\right)$. This shows that all participants had a trend for movement delay, but the participants that used the real task first presented more delay $(\mathrm{m}=530 \mathrm{~ms})$ than the participants who used virtual task first $(\mathrm{m}=303 \mathrm{~ms})$. No further effects were found (Figure $5)$.

Absolute Error- AE

For the AE, there was a significant interaction for Sequence, Interface and Block (F $1,96=164.5, \mathrm{p}<0.001, \mathrm{y} 2=0.63)$. The post-hoc tests show that when individuals 
performed sequence A (virtual task first), the AE increased significantly from A4 at the Keyboard interface $(\mathrm{m}=419 \mathrm{~ms})$ to $\mathrm{T}$ at the Touch screen interface $(\mathrm{m}=661 \mathrm{~ms} \mathrm{md}(\mathrm{CI})=$ $242.0(-361.7,-122.3) ; \mathrm{p}<0.001)$. However, for the individuals who performed sequence $\mathrm{B}$ (real task first), the AE decreased from A4 at the Kinect interface $(\mathrm{m}=947 \mathrm{~ms})$ to $\mathrm{T}$ at the Touch screen interface $(\mathrm{m}=545 \mathrm{~ms} ; \mathrm{md}(\mathrm{CI})=402.7(283.0,522.4)$.

In addition, separate comparisons were made between transfer blocks in order to assess in which sequence the individuals presented better transfer. The post-hoc test found that the transfer block presented a smaller $\mathrm{AE}$ in the sequence B (real first, $\mathrm{m}=804 \mathrm{~ms}$ ) compared to sequence A (virtual first, $\mathrm{m}=656 \mathrm{~ms}$; $\mathrm{md}(\mathrm{CI})=158.5(22.9,294.1)$ ), yet only for ASD group.

Another comparison was made to verify if the first task influenced the initial performance of the second task, and the first acquisition blocks of the same interface were then compared between sequences (for example, A1 Kinect of sequence A versus A1 Kinect of sequence B). The results demonstrated significant differences for the ASD group between blocks A1 for the keyboard interface in sequence A $(\mathrm{m}=475 \mathrm{~ms})$ and sequence B $(\mathrm{m}=718 \mathrm{~ms} ; \mathrm{md}(\mathrm{CI})=-242.8(-375.6,-109.9) ; \mathrm{p}<0.001)$. This difference was not significant for the TD group, nor for the Kinect interface in both groups (Figure 4).

These results suggest that practice with the Kinect interface promoted better transfer of performance to the touch screen interface. However, practice with the keyboard interface did not promote improved transfer to the touch screen interface. In addition, practice in the Kinect interface (more virtual environment) promoted better performance in the keyboard interface (more real environment) but only for people with ASD.

Variable Error - VE

Similar to the AE, there was a significant interaction for Sequence by Interface by Block in the $\operatorname{VE}\left(\mathrm{F}_{1,96}=81.5, \mathrm{p}<0.001, \mathrm{y}^{2}=0.46\right)$. Post-hoc tests showed that the individuals who performed sequence A (virtual task first) when performing the second task at the keyboard interface, VE increased significantly from A4 $(\mathrm{m}=255 \mathrm{~ms})$ to $\mathrm{T}$ in the touch screen task $(m=452 \mathrm{~ms} ; \mathrm{md}(\mathrm{CI})=-196.9(-361.1,-32.7) ; \mathrm{p}=0.019)$. For those who performed sequence B (real task first), the VE decreased from A4 in the Kinect interface $(\mathrm{m}=650 \mathrm{~ms})$ to $\mathrm{T}$ on the touch screen interface $(\mathrm{m}=363 \mathrm{~ms} ; \mathrm{md}(\mathrm{CI})=287.1$ (122.9, 451.3); $\mathrm{p}=0.001)$. 
The regression analysis did not show any significance. This result means that IQ and CARS scores did not have an influence on the improvement in performance ( $\triangle \mathrm{A} 4$ $\mathrm{A} 1)$ in either $\operatorname{AE}\left(\mathrm{F}_{2,30}=0.24, \mathrm{p}=0.788, \mathrm{r}^{2}=0.02\right)$ nor $\mathrm{VE}\left(\mathrm{F}_{2,30}=2.54, \mathrm{p}=0.096, \mathrm{r}^{2}=\right.$ $0.38)$.

\section{DISCUSSION}

The present study investigated the influence of task practice in a real environment (with physical contact - more real characteristics) and virtual (non-physical contact - more virtual characteristics) in people with ASD. In line with our original hypothesis that young people with ASD may perform less well in virtual environments ${ }^{16}$, our findings confirm previous studies that evidenced better performance (both in terms of precision and accuracy) in physical environments when carrying out short term activities in both the ASD and TD group. However, considering higher engagement and enjoyment with using virtual environments, our study is the first to observe that task practice in the virtual environment resulted in an improvement in both accuracy and precision in subsequent practice in the real environment, and this was more pronounced in the ASD group. Another important finding is that people with ASD performed all tasks slower and with more error compared to the TD groups, showing an increased level of difficulty which was particularly apparent in the virtual environment.

Regarding practice of the task (acquisition phase), both the TD and ASD groups who practised the task in the real or virtual interfaces improved performance in the acquisition phase (from the beginning to the end of the practice), demonstrating that both interfaces can be used to promote improvement of performance. Moreover, considering the comparison between tasks, we found that all participants performed better using the keyboard. This observation may be due to greater tactile sensory feedback during real tasks which aids in improving performance ${ }^{32,36}$. Gidley Larson and Mostofsky $(2008)^{44}$ reinforced these findings and showed that people with ASD may have a preference for reliance on proprioceptive information, such as that provided by tactile input, to guide the acquisition of new patterns of movement. Marko et al. (2015) ${ }^{10}$ applied an elementary motor learning task in which reaching movements were perturbed and found that children with ASD performed better compared with healthy controls when learning from feedback proprioceptive errors. 
In addition to possible deficits in proprioception, people with ASD demonstrate more variable patterns of functional difficulties and responsiveness to sensory events ${ }^{49}$. This altered sensory processing (auditory, visual, tactile and movement) is suggested to affect participation in everyday activities including physical activity ${ }^{50,51}$ and the learning of new concepts ${ }^{52}$. Moreover, sensory processing patterns may be associated with preferences for different activities ${ }^{53}$ and the level of difficulty (considering individual experiences). Thus, an increased level of difficulty in tasks due to lower proprioceptive or tactile feedback, altered visual interaction and the higher movement demand from the Kinect interface, may all promote different sensory processing patterns causing difficulty in performing the task.

The observation that participants with ASD performed worse in the environment without physical contact when compared to the physical contact environment can be interpreted considering the "speed and accuracy" trade-off, which proposes that the more difficult the task, the higher the time to complete it (i.e, worse performance during practice). Similar findings have been consistently reported in people with disabilities during a virtual task ${ }^{55-56}$. However, this difficulty in the VR task promoted performance gains, with better execution in the following real task in people with ASD, as demonstrated by the results of both sequences. 1-Sequence A (people with ASD who performed a virtual task first): presented worse performance during VR practice (first practice), and better performance during the second practice on real task (Keyboard), but no transfer to the following different real task (touch screen), and 2- Sequence B (people with ASD who performed a real task first) presented better performance during real practice (first practice) but worse performance during the second practice (virtual task) and better transfer for the following different real task (touch screen). Thus, in summary, this is a significant finding because after a more difficult task (virtual task) the ASD group presented better performance in the following real task (keyboard or touch screen).

Saiano et al. (2015) $)^{27}$ corroborated the present findings by comparing the efficacy of a virtual interface (Kinect) and real interface (gamepad) with an integrated environmentbased approach to teach safety pedestrian skills techniques for adults with ASD. The authors observed that the two interfaces did not show differences in the learning outcome; however, the transfer to real life situations (evaluated by two questionnaires) proved to be more effective in the group that underwent training with Kinect. 
In the comparison of anticipation and delay of movement (constant error), for all phases of the protocol in both sequences, it was observed that all participants presented a tendency to have a delayed response during the task, regardless of the interface; but this delay was observed more often in the virtual task. It can be speculated that the level of difficulty during the accomplishment of the task when using the Kinect was greater, as even the TD group maintained a greater constant error. Chung et al. (2015) ${ }^{57}$ found that for some people with motor skill difficulties, Kinect was less able to detect fine movements due to the system's measurement limitations and therefore questioned whether the system could be used as an accessibility device capable of facilitating voluntary movement of the upper extremities. This idea may justify the tendency for greater delay of movement during the task when using the Kinect since the system is less sensitive at capturing fine movements.

Considering the comparison between ASD and TD groups, regardless of the phase of the protocol, the TD group always maintained a better performance than the ASD group. Moreover, it is also important to emphasise that the TD group reached a potential "ceiling effect" since the beginning of the task, as the results show an excellent performance throughout the protocol. Johnson et al. $(2013)^{58}$ observed that, although the response time was not different between people with ASD and TD, the correlation with manual dexterity and overall motor performance showed that there was a lack of precision during the tasks applied to people with ASD, demonstrating that motor deficits are observable and characterise the symptomatology of these conditions. Studies using a coincident timing task with a control group (TD) and other diseases with altered posture and movement also verified better performance in the control group. Torriani-Pasin et al. (2013) $)^{45}$, De Mello Monteiro et al. (2014 and 2017) $)^{31,32}$ and Bonuzzi et al. (2017) ${ }^{46}$ found that the TD group was more efficient in sequences and errors.

This knowledge is important for multidisciplinary rehabilitation teams to effectively organize an intervention program using technology to enhance learning whilst maintaining enjoyment. As previously proposed by De Mello Monteiro et.al. (2014) ${ }^{32}$, we do not know if this would also be true for other across-task transfers from the virtual to natural environments and after more prolonged training periods. Future work should scrutinise this possibility.

One of the limitations of this study is the lack of information about previous participants computer's keyboard, Kinect and touch screen experience, which could have 
biased the results somehow. A second limitation is that we used different devices and did not analyse specific patterns of movement during practice, as Kinect and Touchscreen were in a vertical position and Keyboard in a horizontal position. Moreover, each device required different ranges of movement. However, the study of Portnoy et al. $(2015)^{59}$ showed that vertical and horizontal digital tasks differ regarding muscle activation patterns, yet this did not affect performance results. We believe that the pattern of movement analysis could provide some interesting results for discussion and is important to be considered for further studies. A third limitation is that we organized a short-term motor learning protocol with only one day of practice and those results could not be generalized as a training protocol, but can be considered as an indication of the influence of Virtual environment to promote better performance to a real task and a long-term protocol should be organized in the future. A fourth limitation is that we could not evaluate the IQ from the control group, and we cannot rule out the possibility that this may have generated speculation about the different results between ASD and TD groups. However, the regression analysis showed no influence of the IQ (and CARS) in the performance for the ASD group, and, it is also important to emphasise that we were very careful to maintain the homogeneity of the groups of comparison (as shown in Table 1), so we can suppose that the difference between groups represents their performance.

Thus, we can conclude that using the task in the current study, people with ASD improved their performance regardless of the stimulated environment, but with worse performance as compared to the TD group. Importantly, the ASD group managed to transfer the practice from a virtual to a real task, which indicates virtual environments may enhance learning of movement in this group. A need for further exploration of its effect across other tasks, training protocol and activities is warranted.

\section{Funding information}

This study was financed in part by the Coordenação de Aperfeiçoamento de Pessoal de Nivel Superior - Brasil (CAPES) - Finance Code 001.

\section{Acknowledgements}


We would like to thank the Integrated psycho-pedagogical support group (GAPI) Special Education School in São Bernardo do Campo, Brazil and to Coordenação de Aperfeiçoamento de Pessoal de Nível Superior, Brazil (CAPES). The authors declare no conflicts of interest.

\section{REFERENCES}

1. Lai, M. C., Lombardo, M. V., \& Baron-Cohen, S. (2014). Autism. Lancet, 383(9920), 896-910. doi: 10.1016/s0140-6736(13)61539-1

2. Association, A. P. (2014). DSM-5: Manual diagnóstico e estatístico de transtornos mentais: Artmed Editora.

3. Hill, D. A., \& Kearley, R. (2013). Autism litigation: outcomes for 2010, trends in decision making and changes in diagnostic criteria. Res Dev Disabil, 34(5), 18431848. doi: 10.1016/j.ridd.2013.02.018

4. Fakhoury, M. (2015). Autistic spectrum disorders: A review of clinical features, theories and diagnosis. Int J Dev Neurosci, 43, 70-77. doi: 10.1016/j.ijdevneu.2015.04.003

5. Rendall, A. R., Truong, D. T., \& Fitch, R. H. (2016). Learning delays in a mouse model of Autism Spectrum Disorder. Behav Brain Res, 303, 201-207. doi: 10.1016/j.bbr.2016.02.006

6. den Brok, W. L., \& Sterkenburg, P. S. (2015). Self-controlled technologies to support skill attainment in persons with an autism spectrum disorder and/or an intellectual disability: a systematic literature review. Disabil Rehabil Assist Technol, 10(1), 1-10. doi: 10.3109/17483107.2014.921248

7. Barbeau, E. B., Meilleur, A. A., Zeffiro, T. A., \& Mottron, L. (2015). Comparing Motor Skills in Autism Spectrum Individuals With and Without Speech Delay. Autism Res. doi: 10.1002/aur.1483

8. Izawa, J., Pekny, S. E., Marko, M. K., Haswell, C. C., Shadmehr, R., \& Mostofsky, S. H. (2012). Motor learning relies on integrated sensory inputs in ADHD, but over-selectively on proprioception in autism spectrum conditions. Autism Res, 5(2), 124-136. doi: 10.1002/aur.1222

9. Stevenson, R. A., Siemann, J. K., Schneider, B. C., Eberly, H. E., Woynaroski, T. G., Camarata, S. M., \& Wallace, M. T. (2014). Multisensory temporal integration in autism spectrum disorders. $J$ Neurosci, 34(3), 691-697. doi: 10.1523/jneurosci.3615-13.2014

10. Marko, M. K., Crocetti, D., Hulst, T., Donchin, O., Shadmehr, R., \& Mostofsky, S. H. (2015). Behavioural and neural basis of anomalous motor learning in children with autism. Brain, 138(Pt 3), 784-797. doi: 10.1093/brain/awu394

11. Xavier, J., Gauthier, S., Cohen, D., Zahoui, M., Chetouani, M., Villa, F., . . . Anzalone, S. (2018). Interpersonal Synchronization, Motor Coordination, and Control Are Impaired During a Dynamic Imitation Task in Children With Autism Spectrum Disorder. Front Psychol, 9, 1467. doi: 10.3389/fpsyg.2018.01467

12. Lindor, E., Rinehart, N., \& Fielding, J. (2018). Distractor Inhibition in Autism Spectrum Disorder: Evidence of a Selective Impairment for Individuals with Co- 
occurring Motor Difficulties. J Autism Dev Disord. doi: 10.1007/s10803-018-37441

13. Miller, H. L., Cacola, P. M., Sherrod, G. M., Patterson, R. M., \& Bugnariu, N. L. (2019). Children with Autism Spectrum Disorder, Developmental Coordination Disorder, and typical development differ in characteristics of dynamic postural control: A preliminary study. Gait Posture, 67, 9-11. doi: 10.1016/j.gaitpost.2018.08.038

14. Kaur, M., S, M. S., \& A, N. B. (2018). Comparing motor performance, praxis, coordination, and interpersonal synchrony between children with and without Autism Spectrum Disorder (ASD). Res Dev Disabil, 72, 79-95. doi: 10.1016/j.ridd.2017.10.025

15. Bryson, S., Garon, N., McMullen, T., Brian, J., Zwaigenbaum, L., Armstrong, V., . . Szatmari, P. (2018). Impaired disengagement of attention and its relationship to emotional distress in infants at high-risk for autism spectrum disorder. J Clin Exp Neuropsychol, 40(5), 487-501. doi: 10.1080/13803395.2017.1372368

16. Herrero, D., Crocetta, T., Massetti, T., de Moraes, I., Trevizan, I., \& Guarnieri, R. (2015). Total Reaction Time Performance of Individuals with Autism after a Virtual Reality Task. IJN an open access journal, 2(1000189), 2376-0281.1000189.

17. Golden, D., \& Getchell, N. (2017). Physical Activity Levels in Children With and Without Autism Spectrum Disorder When Playing Active and Sedentary Xbox Kinect Videogames. Games Health J, 6(2), 97-103. doi: 10.1089/g4h.2016.0083

18. Simoes, M., Bernardes, M., Barros, F., \& Castelo-Branco, M. (2018). Virtual Travel Training for Autism Spectrum Disorder: Proof-of-Concept Interventional Study. JMIR Serious Games, 6(1), e5. doi: 10.2196/games.8428

19. Yang, Y. J. D., Allen, T., Abdullahi, S. M., Pelphrey, K. A., Volkmar, F. R., \& Chapman, S. B. (2018). Neural mechanisms of behavioral change in young adults with high-functioning autism receiving virtual reality social cognition training: A pilot study. Autism Res, 11(5), 713-725. doi: 10.1002/aur.1941

20. Avola, D., Cinque, L., Foresti, G. L., \& Marini, M. R. (2019). An interactive and low-cost full body rehabilitation framework based on 3D immersive serious games. J Biomed Inform, 89, 81-100. doi: 10.1016/j.jbi.2018.11.012

21. Osumi, M., Ichinose, A., Sumitani, M., Wake, N., Sano, Y., Yozu, A., . . Morioka, S. (2017). Restoring movement representation and alleviating phantom limb pain through short-term neurorehabilitation with a virtual reality system. Eur J Pain, 21(1), 140-147. doi: 10.1002/ejp.910

22. Mineo, B. A., Ziegler, W., Gill, S., \& Salkin, D. (2009). Engagement with electronic screen media among students with autism spectrum disorders. $J$ Autism Dev Disord, 39(1), 172-187. doi: 10.1007/s10803-008-0616-0

23. Cox, D. J., Brown, T., Ross, V., Moncrief, M., Schmitt, R., Gaffney, G., \& Reeve, R. (2017). Can Youth with Autism Spectrum Disorder Use Virtual Reality Driving Simulation Training to Evaluate and Improve Driving Performance? An Exploratory Study. J Autism Dev Disord, 47(8), 2544-2555. doi: 10.1007/s10803017-3164-7

24. Patrick, K. E., Hurewitz, F., McCurdy, M. D., Agate, F. T., Daly, B. P., Tarazi, R. A., ... Schultheis, M. T. (2018). Driving Comparisons Between Young Adults with Autism Spectrum Disorder and Typical Development. J Dev Behav Pediatr, 39(6), 451-460. doi: 10.1097/dbp.0000000000000581

25. Ross, V., Cox, D. J., Reeve, R., Brown, T., Moncrief, M., Schmitt, R., \& Gaffney, G. (2018). Measuring the attitudes of novice drivers with autism spectrum disorder 
as an indication of apprehensive driving: Going beyond basic abilities. Autism, 22(1), 62-69. doi: 10.1177/1362361317735959

26. Saiano, M., Garbarino, E., Lumachi, S., Solari, S., \& Sanguineti, V. (2015). Effect of interface type in the VR-based acquisition of pedestrian skills in persons with ASD. Conf Proc IEEE Eng Med Biol Soc, 2015, 5728-5731. doi: 10.1109/embc.2015.7319693

27. Saiano, M., Pellegrino, L., Casadio, M., Summa, S., Garbarino, E., Rossi, V., . . . Sanguineti, V. (2015). Natural interfaces and virtual environments for the acquisition of street crossing and path following skills in adults with Autism Spectrum Disorders: a feasibility study. J Neuroeng Rehabil, 12, 17. doi: 10.1186/s12984-015-0010-z

28. James, G. (1979). The ecological approach to visual perception. Dallas: Houghtom Mifflin.

29. Savelsbergh, G. J., \& Van Der Kamp, J. (2000). Information in learning to coordinate and control movements: Is there a need for specificity of practice? International Journal of Sport Psychology, 31(4), 467-484.

30. van der Weel, F. R., van der Meer, A. L., \& Lee, D. N. (1991). Effect of task on movement control in cerebral palsy: implications for assessment and therapy. Dev Med Child Neurol, 33(5), 419-426.

31. de Mello Monteiro, C. B., da Silva, T. D., de Abreu, L. C., Fregni, F., de Araujo, L. V., Ferreira, F., \& Leone, C. (2017). Short-term motor learning through nonimmersive virtual reality task in individuals with down syndrome. BMC Neurol, 17(1), 71. doi: 10.1186/s12883-017-0852-z

32. de Mello Monteiro, C. B., Massetti, T., da Silva, T. D., van der Kamp, J., de Abreu, L. C., Leone, C., \& Savelsbergh, G. J. (2014). Transfer of motor learning from virtual to natural environments in individuals with cerebral palsy. Research in developmental disabilities, 35(10), 2430-2437.

33. Holm, R., \& Priglinger, M. (2008). Virtual Reality Training As A Method For Interactive And Experience-based Learning. Paper presented at the Intelligent Energy Conference and Exhibition.

34. Spence, C. (2003). Crossmodal attention and multisensory integration: implications for multimodal interface design. Paper presented at the Proceedings of the 5th international conference on Multimodal interfaces.

35. Yano, H., Ogi, T., \& Hirose, M. (1998). Development of haptic suit for whole human body using vibrators. Trans. of the Virtual Reality Society of Japan, 3(3), 141-148.

36. Martins, F. P. A., Massetti, T., Crocetta, T. B., Lopes, P. B., da Silva, A. A., Figueiredo, E. F., ... \& de Mello Monteiro, C. B. (2019). Analysis of motor performance in individuals with cerebral palsy using a non-immersive virtual reality task-a pilot study. Neuropsychiatric disease and treatment, 15, 417.

37. Al Backer, N. B. (2016). Correlation between Autism Treatment Evaluation Checklist (ATEC) and Childhood Autism Rating Scale (CARS) in the evaluation of autism spectrum disorder. Sudan J Paediatr, 16(1), 17-22.

38. Campbell, J. M. (1998). Internal and external validity of seven Wechsler Intelligence Scale for Children-Third Edition short forms in a sample of psychiatric inpatients. Psychological Assessment, 10(4), 431.

39. Yu, T. Y., Chou, W., Chow, J. C., Lin, C. H., Tung, L. C., \& Chen, K. L. (2018). IQ discrepancy differentiates levels of fine motor skills and their relationship in children with autism spectrum disorders. Neuropsychiatr Dis Treat, 14, 597-605. 
doi: $10.2147 /$ ndt.s153102

40. Crocetta, T. B., de Araújo, L. V., Guarnieri, R., Massetti, T., Ferreira, F. H. I. B., de Abreu, L. C., \& de Mello Monteiro, C. B. (2018). Virtual reality software package for implementing motor learning and rehabilitation experiments. Virtual Reality, 22(3), 199-209.

41. Kim, R., Nauhaus, G., Glazek, K., Young, D., \& Lin, S. (2013). Development of coincidence-anticipation timing in a catching task. Percept Mot Skills, 117(1), 1361-1380.

42. Malheiros, S. R., da Silva, T. D., Favero, F. M., de Abreu, L. C., Fregni, F., Ribeiro, D. C., \& de Mello Monteiro, C. B. (2016). Computer task performance by subjects with Duchenne muscular dystrophy. Neuropsychiatr Dis Treat, 12, 41-48. doi: $10.2147 /$ ndt.s87735

43. Trevizan, I. L., Silva, T. D., Dawes, H., Massetti, T., Crocetta, T. B., Favero, F. M., ... Monteiro, C. B. M. (2018). Efficacy of different interaction devices using nonimmersive virtual tasks in individuals with Amyotrophic Lateral Sclerosis: a crosssectional randomized trial. BMC Neurol, 18(1), 209. doi: 10.1186/s12883-0181212-3

44. Gidley Larson, J. C., \& Mostofsky, S. H. (2008). Evidence that the pattern of visuomotor sequence learning is altered in children with autism. Autism Res, 1(6), 341-353. doi: 10.1002/aur.54

45. Torriani-Pasin, C., Bonuzzi, G. M., Soares, M. A., Antunes, G. L., Palma, G. C., Monteiro, C. B., . . . Correa, U. C. (2013). Performance of Down syndrome subjects during a coincident timing task. Int Arch Med, 6(1), 15. doi: 10.1186/17557682-6-15

46. Bonuzzi, G. M. G., Corrêa, U. C., Antunes, G. L., Monteiro, C. B. d. M., Júnior, A. P., Freudenheim, A. M., \& Torriani-Pasin, C. (2016). Effects of the task complexity on the performance of a Coincidence Timing Task of people with Down syndrome. Motricidade, 12(4), 15-23.

47. Bezerra, I. M. P., Crocetta, T. B., Massetti, T., Silva, T. D. D., Guarnieri, R., Meira, C. M., Jr., . . Monteiro, C. B. M. (2018). Functional performance comparison between real and virtual tasks in older adults: A cross-sectional study. Medicine (Baltimore), 97(4), e9612. doi: 10.1097/md.0000000000009612

48. Silva-Filho, E., Okano, A. H., Morya, E., Albuquerque, J., Cacho, E., Unal, G., . Pegado, R. (2018). Neuromodulation treats Chikungunya arthralgia: a randomized controlled trial. Sci Rep, 8(1), 16010. doi: 10.1038/s41598-018-34514-4

49. Kientz, M. A., \& Dunn, W. (1997). A comparison of the performance of children with and without autism on the Sensory Profile. Am J Occup Ther, 51(7), 530-537.

50. Lawson, L. M., \& Foster, L. (2016). Sensory Patterns, Obesity, and Physical Activity Participation of Children With Autism Spectrum Disorder. Am J Occup Ther, 70(5), 7005180070p7005180071-7005180078. doi: 10.5014/ajot.2016.021535

51. Little, L. M., Dean, E., Tomchek, S., \& Dunn, W. (2018). Sensory Processing Patterns in Autism, Attention Deficit Hyperactivity Disorder, and Typical Development. Phys Occup Ther Pediatr, 38(3), 243-254. doi: 10.1080/01942638.2017.1390809

52. Brown, N. B., \& Dunn, W. (2010). Relationship between context and sensory processing in children with autism. Am J Occup Ther, 64(3), 474-483.

53. Ismael, N. T., Lawson, L. A., \& Cox, J. A. (2015). The relationship between children's sensory processing patterns and their leisure preferences and 
participation patterns. Can J Occup Ther, 82(5), 316-324. doi:

10.1177/0008417415577421

54. Fernani, D., Prado, M. T. A., da Silva, T. D., Massetti, T., de Abreu, L. C., Magalhaes, F. H., ... de Mello Monteiro, C. B. (2017). Evaluation of speedaccuracy trade-off in a computer task in individuals with cerebral palsy: a crosssectional study. BMC Neurol, 17(1), 143. doi: 10.1186/s12883-017-0920-4

55. Stevens, D., Anderson, D. I., O'Dwyer, N. J., \& Mark Williams, A. (2012). Does self-efficacy mediate transfer effects in the learning of easy and difficult motor skills? Conscious Cogn, 21(3), 1122-1128. doi: 10.1016/j.concog.2012.03.014

56. Robert, M. T., \& Levin, M. F. (2018). Validation of reaching in a virtual environment in typically developing children and children with mild unilateral cerebral palsy. Dev Med Child Neurol, 60(4), 382-390. doi: 10.1111/dmcn.13688

57. Chung, P. J., Vanderbilt, D. L., Schrager, S. M., Nguyen, E., \& Fowler, E. (2015). Active Videogaming for Individuals with Severe Movement Disorders: Results from a Community Study. Games Health J, 4(3), 190-194. doi: 10.1089/g4h.2014.0091

58. Johnson, B. P., Rinehart, N. J., White, O., Millist, L., \& Fielding, J. (2013). Saccade adaptation in autism and Asperger's disorder. Neuroscience, 243, 76-87. doi: 10.1016/j.neuroscience.2013.03.051

59. Portnoy, S., Rosenberg, L., Alazraki, T., Elyakim, E., \& Friedman, J. (2015). Differences in muscle activity patterns and graphical product quality in children copying and tracing activities on horizontal or vertical surfaces. J Electromyogr Kinesiol, 25(3), 540-547. doi: 10.1016/j.jelekin.2015.01.011

\section{LEGENDS OF FIGURES}

Figure 1. Representative design of the accomplishment of the coincident timing task in the use of the kinect interface.

A. Demonstration of error performed by the participant on each attempt (red light - unsuccessful). B. Demonstration of hit performed by the participant on each attempt (green light - successful). Main image is about an example of a participant performing the task on Kinect. 1. Performing the task on the touch screen interface. 2. Performing the task on the keyboard interface.

Figure 2. Experimental design.

TD: typical development; ASD: autism spectrum disorder.

Figure 3. Constant error for all phases of the study by both groups, sequences and interfaces (mean and standard error).

K: kinect; KB: keyboard; TS: touch screen; TD: typical development; ASD: autism spectrum disorder; A: acquisition phase blocks (1 through 4$)$; R: retention phase; T: transfer phase. 
Figure 4. Absolute error for all phases of the study by both groups, sequences and interfaces (mean and standard error).

K: kinect; KB: keyboard; TS: touch screen; TD: typical development; ASD: autism spectrum disorder; A: acquisition phase blocks (1 through 4$)$; R: retention phase; T: transfer phase.

Figure 5. Variable error for all phases of the study on both groups, sequences, and interfaces (mean and standard error).

K: kinect; KB: keyboard; TS: touch screen; TD: typical development; ASD: autism spectrum disorder; A: acquisition phase blocks (1 through 4); R: retention phase; $\mathrm{T}$ : transfer phase.

Table 1. Demographic data and comparisons for each of the subgroups.

ASD: group with autism spectrum disorder; TD: group with typical development; ASD-A and TD-A: groups that started with Kinect; ASD-B and TD-B: groups that started with Keyboard; M: mean; SD: standard deviation; min: minimum value; max: maximum value; md: mean difference; 1 : lower bound of the Confidence Interval (95\%) of the difference; u: upper bound of the Confidence Interval $(95 \%)$ of the difference; n: sample size; BMI: Body Mass Index; IQ: Intelligence Quotient; CARS: Childhood Autism Rating Scale. *MANOVA was carried out in order to find out differences ASD and TD groups and interface subgroups (A and B) with Least Significance Difference as post-hoc test;** values presented regarding posthoc tests; $\dagger$ independent samples t-test comparing subgroups A and B; §chi-square test, probably the sample size was small to find significant results. 